\title{
Differences in the Learning Principles Dominating Student-Student vs. Student-Instructor Interactions while Working on Programming Tasks
}

\author{
Alessio Gaspar \\ Department of \\ Computer Science \& \\ Engineering \\ University of South \\ Florida \\ 813-974-2932 \\ alessio@usf.edu
}

\author{
Joni Torsella \\ \& Nora Honken \\ Department of \\ Engineering Education \\ University of Cincinnati \\ 513-556-1224 \\ joni.torsella@uc.edu \\ honkenna@ucmail.uc.edu
}

\author{
Sohum Sohoni \\ School of Computing \\ Informatics and Decision \\ Systems Engineering \\ Arizona State University \\ 480-727-1590 \\ sohum.sohoni@asu.edu
}

\author{
Colin Arnold \\ Department of \\ Computer Science \& \\ Engineering \\ University of South \\ Florida \\ 813-974-3780 \\ cdarnold@usf.edu
}

\begin{abstract}
Peer learning principles have been successfully applied to novice programmers. Pedagogies such as Pair Programming, Peer Testing, Peer review of code or tests, or, more generally Peer Instruction, have repeatedly demonstrated their effectiveness in improving both individual performance and retention rates.

This paper proposes to supplement the existing literature by investigating how students interact with one another during collaborative programming tasks. More specifically, we are interested in comparing the learning principles used during student-student interactions with those used during studentinstructor or student-teaching assistant dialogs.

Students in online and face to face courses, who worked collaboratively on programming assignments, were surveyed to gain an understanding of the frequency with which they engaged in specific activities. These that are representative of the learning principles that have been supported by research to promote learning.

Results suggest that some learning principles, may be absent from student-student interactions. We discuss how the success of collaborative programming pedagogies put into question the role of these principles and whether they may contribute to further improve peer-based approaches.
\end{abstract}

\section{Keywords}

Novice Programmers; Peer Learning; Learning Principles

\section{INTRODUCTION}

\subsection{Motivation}

Active learning and constructivist pedagogies have paved the way for computer science educators to transition from being "Sages on the stage" to being "Guides on the side"; e.g. [1, 2]. Among them, pedagogies based on peer learning such as peer instruction $[3,4]$, pair programming $[5,6]$ and peer testing $[\mathbf{7}, \mathbf{8}]$ have been

Permission to make digital or hard copies of all or part of this work for personal or classroom use is granted without fee provided that copies are not made or distributed for profit or commercial advantage and that copies bear this notice and the full citation on the first page. Copyrights for components of this work owned by others than ACM must be honored. Abstracting with credit is permitted. To copy otherwise, or republish, to post on servers or to redistribute to lists, requires prior specific permission and/or a fee. Request permissions from Permissions@acm.org.

SIGCSE'16, March 2-5, 2016, Memphis, TN, USA.

(C) 2016 ACM. ISBN 978-1-4503-3685-7/16/03 ...\$15.00.

DOI: http://dx.doi.org/10.1145/2839509.2844627 repeatedly reported as successful at improving student grades, retention, motivation and individual programming skills.

This success invites researchers to further investigate the nature of the didactic dialog which students establish with one another, and whether this dialog is related to learning principles that have been proven to be beneficial. Also of interest is how similar, or different, student-student interactions are from those between students and instructors or teaching assistant. Gaining insights on this has potential to help us better understand why collaborative programming activities are so beneficial to students.

This research is further motivated by the growth of online programs. Within the context of the previous research question, we also want to assess whether our findings are stable when switching from face-to-face to online delivery modality. Means et al. [9] state that solid empirical research on implementations of online learning is limited, and is lagging behind technological innovations that act as game-changers. Although a body of knowledge exists on teaching and learning online, researchers are discovering that different aspects of online learning need to be explored in more detail within specific contexts ([9], p. 37).

\subsection{Specific Objective of this Study}

This paper is a first step towards investigating the specifics of how students help one another while working on programming tasks. Unlike previous studies, it is the didactic nature of the interactions that was examined, rather than measurements of the impact collaborating has on improving grades, individual skills, motivation, or retention rate in the discipline.

While the long term goals of this project are to understand the impact of the didactic exchanges among students, and to drive new pedagogies based on these findings, this paper is an initial necessary step in this direction. Based on Fincher and Petre's method of science approach [10] p.16, this paper may be viewed as a step towards developing an explanatory theory preliminary to a predictive one. The data collection and analysis we leveraged in this study is consistent with this phase of a theory's development.

\subsection{Conceptual Framework}

In 2008 a group of researchers, brought together by the Association of Psychological Sciences and the American Psychological Association, accumulated a list of learning principles that had been proven through research to lead to learning. Their purpose was to disseminate the list to practitioners with the hope of improving learning in schools [11]. We reasoned 
that if learning actually takes place during collaborative programming assignments that some of the principles should be present. After reviewing the 25 Learning Principles, we felt that deep questioning and desired difficulty had a strong potential to support collaborative programming activities. As such, we decided to focus our first study on these.

The first principle, Deep Questioning, is related to asking why, how, what-if questions. These types of questions have been shown to promote deeper comprehension and learning at a deeper level $[11,12]$. Collaborative work creates opportunities for students to ask each other such questions. It also enable them to treat different solutions emerging in the group as what if scenarios they may justify to one another.

The second principle we investigated is Desirable Difficulty. It involves the learner having to exert effort during acquisition and retrieval of information. It has been shown that, when students have to organize their thoughts on a topic, learning is enhanced [11]. This learning principle is relevant to collaborative programming scenarios in so far that participants are not necessarily allowed to indulge in a passive pupil role. Instead, they are expected to contribute equally by their peers. The latter implies investing efforts in understanding explanations from other members who are not solely dedicated to help them.

Due to their established relevance to computing education [1, 2, 13], we also considered constructivism and instructivism. Constructivists theorize that learners construct their knowledge through experiences, while instructivists theorize that an instructor is at the center of learning and are responsible to pass on their knowledge to the student. In collaborative programming tasks, we hypothesize that one participant rarely assumes a dedicated teacher role. While it is easy to assume that this entails a form of constructivism, no study has, to the best of our knowledge, characterized student-student interactions as such. As an extension of the above, we also considered the pupil's independence as a way to identify passive learning or instructivist pedagogies.

Last but not least, we then supplemented the above via the concepts of knowledge transfer sequences vs. collaborative sequences. In [14], Haller et al. use conversation analysis as a method to understand how students learn from each other in cooperative groups. Although the study was conducted in a chemical engineering class, it has implications in the broad context of science and engineering courses using teamwork. The authors classify the conversations into two kinds of teachinglearning interactions, transfer of knowledge sequences, and collaborative sequences. In the former, the students engage in a teacher-pupil dialog but with a dialogic feedback loop that is usually not present in a classroom. In the collaborative sequences, learning is accomplished through shared thinking, and knowledgebuilding through discussion.

The above principles and pedagogies served as the framework of our study and dictated the design of a survey instrument.

\subsection{Hypothesis}

This study aims at gaining insights about how students learn while working on programming tasks together. More specifically, we seek to establish whether, and how, student-student didactic interactions differ from those they have with instructors or teaching assistants. Our hypothesis is that the nature of both didactic dialogs should reflect the focus of instructors and teaching assistant to support their interlocutor's learning, while peer might be more focused on completing assignments successfully. Differences in the interactions themselves would suggest that, while successful, student-student interactions may be further improved by integrating elements from educational practices which have already demonstrated their effectiveness in non-peer learning settings.

\subsection{Terminology}

In the remainder of this paper, "Programming tasks" will encompass both graded and non-graded programming activities. "Working together" will describe dynamics ranging from informal study groups, to supervised activities; e.g. pair programming or team assignments. "Student-instructor" will refer to interactions between students and either faculty or teaching assistants (TA).

\section{METHODS}

Given our objectives, our primary source of information is the students themselves. We therefore adopted a cross-sectional study research design using an online anonymous survey administered in Survey Monkey to students at three different universities.

\subsection{Overall Survey Design \& Organization}

The survey was designed to gather the same information in two distinct scenarios; when the respondents are working with other students, and when they are working with instructors or teaching assistants. As a result, the survey was divided in two parts respectively dedicated to each scenario. In order to keep responses comparable, we used the same questions in both, albeit after adapting the phrasing to fit the specifics of the scenario.

We also used three "pivot" questions. These allowed us to determine whether a responded had indeed participated in collaborative programming activities with other students or instructors / teaching assistants. Based on their responses to these questions, we skipped the parts of the survey focused on interactions they didn't experience personally.

The core questions of our survey, which are detailed in the following subsections, allowed respondents to express the frequency with which they engaged in various behaviors. These frequency levels were expressed via 5-point Likert items ranging from almost never (1) to most of the time (5). During analysis, the two lowest levels were aggregated into a nominal category seldom. Similarly, the two highest level 3 was kept as sometimes, and level 4 and 5 were aggregated into often.

\subsection{Types of Assistance Received}

Each of the two main parts of the survey started with a question about how often respondents experienced specific scenarios when requesting help in programming tasks.

You have problems implementing a part of the project and
you ask_for help. Rate the frequency with which each of
the following situations occurs.
1. The tells me exactly how to fix it without fixing the
problems for me
2. The_fixes the problems for me without explaining
3. The directs me to the part of the lecture I
misunderstood without fixing the problems for me
4. The and I work together and both contribute
5. The the fix the problems
6. The explains what is wrong but not how to fix it and
does not fix the problems for me

The above template was used as $\mathbf{Q 7}$ in the first part of the survey and Q12 in the second. The blanks were respectively replaced with "another student" and "the instructor or teaching assistant". Based on our conceptual framework, the six items were carefully phrased. Table 1 shows which items are related to scenarios 
illustrating either high or low independence on the part of the pupil - i.e. the respondent. It also shows which scenarios reveal teaching-learning interactions based on knowledge transfer sequences, collaborative sequences, and one scenario is used to capture situations where such sequences are absent; item \#2.

Table 1 - Learning Principles Captured in Q7 / Q12

\begin{tabular}{|l|l|l|}
\hline \multicolumn{2}{|c|}{ Learning Principles } & \multicolumn{1}{c|}{ Items } \\
\hline \multirow{2}{*}{ Pupils' Independence } & High & $3,4,6$ \\
\cline { 2 - 3 } & Low & $1,2,5$ \\
\hline \multirow{2}{*}{$\begin{array}{l}\text { Teaching-Learning } \\
\text { Interactions }\end{array}$} & KT-Sequence & $1,2,5,6$ \\
\cline { 2 - 3 } & C-Sequence & 4 \\
\cline { 2 - 3 } & None & 2 \\
\hline
\end{tabular}

\subsection{Types of Explanations Received}

Last, we used another frequency level question in each part of the survey to gain more detailed insights regarding the nature of the explanations received by respondents when they asked for help.

You have problems implementing a part of the project and you ask __for help. Rate the frequency with which each of the following situations occurs.

1. Their explanations take into consideration my mistakes or misunderstandings

2. Their explanations relate other learning situations or concepts I encountered

3. Their explanations only describe the steps to solve the problem

4. Their explanations take into consideration my current understanding or knowledge

5. Their explanations include what-if scenarios

6. Their explanations require me to put effort into understanding them

7. Their explanations focus on why a piece of code works

8. Their explanations focus on how a piece of code works

The above template was used as Q9 in the first part of the survey and Q14 in the second. The blanks were respectively replaced with "another student" and "the instructor or teaching assistant".

Table 2 - Learning Principles Captured in Q9 / Q14

\begin{tabular}{|l|l|}
\hline \multicolumn{1}{|c|}{ Learning Principles } & \multicolumn{1}{c|}{ Items } \\
\hline Deep Questioning & $5,7,8$ \\
\hline Desirable Difficulty & 6 \\
\hline Constructivism & $1,2,4$ \\
\hline Instructivism & 3 \\
\hline
\end{tabular}

Table 2 shows how each item in these questions was phrased to relate to the two previously selected learning principles, i.e. Deep Questioning and Desirable Difficulty. We also phrased items so that some would also relate to Instructivism or Constructivism.

\subsection{Population \& Sample}

The survey was administered using surveymonkey. Instructors disseminated the URL to their students at the end of the spring 2015 semester. Participation was entirely optional, with no additional incentive provided. The survey was anonymous, not gathering any identifying or even demographic information about respondents. The sample was therefore self-selected solely on the basis of respondents' inclination to volunteer for this study. No questions were mandatory, allowing respondents to skip questions for which they didn't feel they had a meaningful or relevant response. This resulted in a variable number of responses for different questions, as noted during our data analysis.

The surveyed population consisted of engineering undergraduate students in US institutions, all enrolled in offerings with a strong programming requirement. Four of the offerings in which the study was conducted were taught in an online Information Technology BS degree in a research-intensive university; "Object Oriented Programming", "Programming Concepts", "IT Program Design" and "Data Structures". These span the sophomore to senior years and rely on the $\mathrm{C}, \mathrm{C}++$ and Java programming languages. Students are assigned programming assignments and interact with the instructor via emails, forums posts and weekly, optional, "live" online sessions. The latter are organized in a flipped manner since the lecture material is already conveyed via reading assignments or recorded videos. Most enrolled students are adults, work full time, and value the flexibility afforded by online delivery.

"Systems Fundamentals" was also offered online. It covers number systems and arithmetic, Boolean algebra, logic gates, truth tables, finite state machines, and logic circuits. A significant portion of the grade is on individual and team projects where students implement simple circuits in behavioral Verilog; e.g. counters, simple ALU's.

"Microprocessors" was delivered as both a face-to-face and a separate online offering. It covers microcomputer architecture, instruction set, assembly language programming, I/O and memory interfacing, and exception/interrupt handling. A significant portion of the grade is based on individual and team projects which involve programming tasks in MIPS-like assembly.

The online versions of the Systems Fundamentals and the Microprocessor course were offered as part of a fully-online Software Engineering BS degree in a research-intensive university. The Systems Fundamentals course was also taken by students in a fully-online Information Technology BS degree. Both courses saw a distribution of students in all four years, with a majority of sophomores and juniors.

"Models II", a required freshman course, was taught face-to-face in a department of Engineering Education in a research-intensive university. The course focusses on programming with MATLAB ${ }^{\circledR}$. The overwhelming majority of the students in this course were traditional college students. Multiple sections, taught by different instructors, were surveyed.

\subsection{Analysis}

Data was analyzed using the Minitab software. Descriptive statistics were calculated and Chi Square Goodness of Fit tests were run to determine if the distribution of responses was different between online and face to face courses.

\section{RESULTS}

\subsection{Response Rates}

The survey was disseminated to 479 students in the six online classes and 478 in the four face-to-face ones. Respective response rates were 17\% (81 responses) and 11\% (51). Overall, we had 132 respondents for a $14 \%$ response rate.

\subsection{Identifying Dominant Scenarios}

Table 5 and Table 6 show the raw data that was collected during this study. Each table respectively provides the data about one of two pairs of questions.

Table 5 summarizes the data related to the types of assistance received (questions $\mathbf{Q 7}$ and $\mathbf{Q 1 2}$ ). Table 6 does the same for the 
pair of questions related to the types of explanations (Q9 and Q14). A Chi-Square test was used to examine the significance of relationships between our two nominal level variables: class type and level of frequency. Class type includes two categories: faceto-face courses - F2F - and online courses - ONL. Level of frequency includes seldom, sometimes and often.

In each table, the first question focuses on student-student interactions, while the second focuses on student-instructor ones. Each table lists the response items used in both questions of the corresponding pair. We are interested in the number of responses in each of our categories.

Our goal is to compare student-student interactions with studentinstructors ones. To this end, we need to characterize how the distribution of responses, both ONL and F2F, varies when comparing the first question of the pair to the second. Let us detail the process we used to do so by considering an example.

Questions Q7 and Q12 both refer to the types of assistance received respectively in student-student and student-instructor interactions. Item \#4 describes a scenario where both the student, and the person they are working with, contribute equally to solving the problems encountered.

Table 5 identifies the nominal category with the most respondents, for this item.

For face-to-face students;

- $\quad$ For Q7, the mode is in the often category (22 as opposed to 9 for seldom and 14 for sometimes).

- For Q12, the mode is in sometimes (15 as opposed to 11 for often and 10 for seldom).

Thus in a F2F situation, it is more common to have equal contribution to solving a problem when students are collaborating. For online students;

- $\quad$ In Q7, the mode is in seldom (15 vs. 10 or 7).

- In Q12, often is the least popular response while sometimes and seldom are really close (resp. 6 and 5 respondents).

In an online situation equal contribution is more frequently noted when the interaction is between the student and TA/instructor, although it is not dominantly frequent even there.

This example illustrates that a given scenario might be slightly more frequently reported in one of the two types of interactions, while still being globally infrequent. To identify such a situation, we then look at whether the mode in one of the questions is not in the often category. We do so for both ONL and F2F students. In the above example;

- We first look at face-to-face students (F2F) in studentstudent interactions $(\mathbf{Q 7 )}$. The often responses dominate. For online students (ONL), it fails to clearly dominate.

- We then look at student-instructor interactions (Q12) and notice that, for both face-to-face and online students, the dominant number of responses is not in often.

Therefore, it seems that item \#4's scenario is less frequent in student-instructor interactions than student-student ones.

\section{DISCUSSION}

Table 3 shows the result of applying this process to all items from questions Q7 and Q12. Table 4 shows similar data for questions Q9 and Q14. In some instance, the item was not found to dominate in either type of interactions $(=)$. The last column revealed that, most of the time, both student-student and studentinstructor interactions did not feature a mode in the often category for either online or face-to-face students ("both").

The following subsections respectively review the summary tables related to the types of assistance and the types of explanations.

\subsection{Differences in Types of Assistance}

We first applied the above-defined process to the data in Table 5. Questions Q7 and Q12 prompted respondents to estimate the frequency of six scenarios each capturing specific learning principles. Table 3 shows the resulting summary information. Reviewing the survey item results in

Table 5 individually, we discover some interesting results. Several practices identified by our question items clearly do not dominate student behavior; e.g. strategies whereby students are provided step-by-step ways to fix their problems, or even worse, have their problems fixed without having to solve issues themselves. These practices are weak in both face-to-face vs. online, along with, student-student vs student-instructor scenarios. These observations are actually positive, as such practices do not align with what educators recognize as efficient in teaching.

Table 3 - Q7 / Q12 - Summary Comparison

\begin{tabular}{|c|c|c|c|c|}
\hline \multirow{2}{*}{$\begin{array}{c}\text { Item } \\
\#\end{array}$} & \multicolumn{3}{|c|}{ Student-student vs. Student-instructor } \\
\cline { 2 - 5 } & $\begin{array}{c}\text { Most frequent } \\
\text { F2F }\end{array}$ & $\begin{array}{c}\text { Most frequent } \\
\text { ONL }\end{array}$ & \multicolumn{2}{c|}{ Mode is in "often" } \\
\cline { 4 - 5 } & Student & $=$ & Student & Instructor \\
\hline 1 & $=$ & $=$ & NO & NO \\
\hline 2 & $=$ & Instructor & NO & NO \\
\hline 3 & $=$ & Instructor & & NO \\
\hline 4 & Student & $=$ & NO & NO \\
\hline 5 & $=$ & Instructor & NO & \\
\hline 6 & Instructor & \multicolumn{2}{c}{} \\
\hline
\end{tabular}

Similarly, the practice of providing help by referring the student to study material, e.g. lectures, without further help is not dominant in any of the scenarios either. This might actually suggest an improvement to both face-to-face and online delivery approaches; as well as, student-student vs. student-instructor didactic dialogs. Assuming that pointing students to revisit relevant study material, given the specific difficulties they are encountering, is an instance of the Desirable Difficulty principle, then this result suggests that we might want to better prepare instructors and students alike.

Reviewing the results as they pertain to our defined learning principle pattern, we find the responses to the three items corresponding to pupil high independence (3,4 and 6) show a strong dominance for instructors in online settings, and a mixed dominance in face-to-face ones. The responses to the three items corresponding to pupil low independence (1,2 and 5) do not show any clear dominance in either setting or interaction type with the highest dominant vote being sometimes for item 5; thus neither students nor instructors are providing answers without explanation. Therefore we find that, in online settings, instructors are more likely to use constructivist techniques. Further, due to the mixed results for student-student interaction in both scenarios we see that students would potentially benefit from being taught some learning strategies to reap the benefits seen in studentinstructor dialogs.

The responses to the question corresponding to collaborative sequences (item 4) suggest that face-to-face students often work together sharing knowledge and discussing problems to be able to learn, and to a lesser extent, with instructors. However, in online settings we find that students seldom have such conversations with either other students or instructors. Not surprisingly, we find that students seldom engage in a knowledge transfer sequence when working with other students. However, we find mixed results in students-instructors interactions. In face-to-face settings, we find that instructors explain how to fix the problem while 
explaining why it was wrong. In online settings, instructors more often explain what is wrong but not how to fix it. Lastly, we sometimes find instructors who, regardless of the setting, fix the problem without explanation.

\subsection{Differences in Types of Explanations}

We then applied the same process to the data provided in Table 6 and obtained the summary presented in Table 4 . The responses to the item representing desirable difficulty suggest that this learning principle is less often leveraged when students work with one another than when they work with an instructor.

The three items representing the deep questioning learning principles revealed that this practice occurs more frequently when students work with the instructor than with peers. Similarly, it occurs more frequently in face-to-face than online settings. However, results also show that the number of responses in the often frequency level do not represent a majority of respondents in any scenario; i.e. student-student vs. student-instructor, or face-toface vs. online. This suggests that this learning principle might be particularly important to introduce to both instructor and students.

Table 4 - Q9 / Q14 - Summary Comparison

\begin{tabular}{|c|c|c|c|c|}
\hline \multirow{2}{*}{$\begin{array}{c}\text { Item } \\
\#\end{array}$} & \multicolumn{4}{|c|}{ Student-student vs. Student-instructor } \\
\cline { 3 - 5 } & $\begin{array}{c}\text { Most frequent } \\
\text { F2F }\end{array}$ & $\begin{array}{c}\text { Most frequent } \\
\text { ONL }\end{array}$ & \multicolumn{2}{|c|}{ Mode is in "often" for... } \\
\cline { 4 - 5 } & Instructor & Instructor & & \\
\hline 1 & Student & Instructor \\
\hline 2 & Instructor & Instructor & NO & \\
\hline 3 & Instructor & Instructor & NO & NO \\
\hline 4 & Instructor & $=$ & NO & \\
\hline 5 & $=$ & Instructor & NO & NO \\
\hline 6 & Instructor & Instructor & & \\
\hline 7 & $=$ & Instructor & & \\
\hline 8 & Instructor & Instructor & NO & NO \\
\hline
\end{tabular}

The three items representing constructivist learning principles led to similar results with respect to when they occur most frequently. However, only student-student and online showed a lack of dominance for the often level, thus narrowing down the scenarios in which remediation should be applied.

The item capturing an instructivist pedagogy was, interestingly, more frequent in face-to-face settings and when working with the instructor. However, it also revealed that none of the scenarios, i.e. student-student vs student-instructor and face-to-face vs. online, featured a dominant proportion of respondents in the often level.

\subsection{Threats to validity}

The anonymous nature of the survey, its online administration and the lack of any added incentive to participate, provided both potentially positive and negative effects on the results.

This setting helped ensure that respondents shared their genuine opinions by mitigating biases based on perceptions of what would be "ideal responses". The lack of incentive prevented random responses provided just to get to the reward.

However, voluntary participation always invites the so-called selfselection sampling bias. Those who are motivated to respond, often do so under strong opinions, whether positive or negative. In the same line of thought, we ensured that the survey would randomize items in questions in order to prevent biases induced by arbitrary response patterns.

While our sample size allowed us to draw statistically significant conclusions, the response rates were low. It is reasonable to assume that the lack of incentive is at least partially responsible.
However, it is difficult to assess whether the low response rates might have been more detrimental than the various biases enabled by incentives. The anonymity of the survey prevented us from supplementing our analysis with a profiling of the nonrespondents, which may have helped characterize respondents' motivations. In subsequent studies, we will seek funding to attempt to incentivize participation. However, we will do so using an option for students to skip responding and just get the incentive when they open the survey. We previously used this technique to mitigate random responses provided by respondents who are solely incentive-driven.

Another potential threat lies in the nature of online asynchronous courses. In such courses, the nature of student interactions is much more diversified and less easy to trace; e.g. emails, web-meetings, face-to-face study groups, in-lab supervised collaborative work... This is not taken into account in this study.

\section{Conclusions \& Future work}

The results from this pilot study merit a more comprehensive investigation of the didactic dialog students establish with each other. This next step of our study will be conducted in partnership with additional faculty who are invited to contact us to express an interest in participating in a broader study on this topic.

We plan on using the results of such a larger-scale validation study to provide foundations for modifying peer learning interventions in order to lead students to further minimize interactions in which they provide each other with "too much" information which results in reducing the pupil's independence.

Once we have gathered sufficient quantitative data, we plan on integrating more qualitative research designs to help us understand why students rely on the specific type of didactic interactions the quantitative study identified. This will involve explaining the observed behaviors through focus group, interviews, and other qualitative approaches such as linguistic analysis of student dialog transcribed from video recording of work sessions.

\section{ACKNOWLEDGMENTS}

This material is based in part upon work supported by the National Science Foundation under award \#0836863 Any opinions, findings, and conclusions or recommendations expressed in this publication are those of the author(s) and do not necessarily reflect the views of the National Science Foundation.

The authors are also indebted to Drs. Kastner, and Bucks from the University of Cincinnati for kindly disseminating the link to the online survey to their "Models II" students.

\section{REFERENCES}

[1] T. Wulf. 2005. Constructivist approaches for teaching computer programming. In Proceedings of the 6th conference on Information technology education (SIGITE '05). ACM, New York, NY, USA, 245-248.

[2] A.K. Lui, R. Kwan, M. Poon, and Y.H.Y. Cheung. 2004. Saving weak programming students: applying constructivism in a first programming course. SIGCSE Bull. 36:2, 72-76.

[3] B. Simon, J. Parris, and J. Spacc. 2013. How we teach impacts student learning: peer instruction vs. lecture in CS0. In Proceeding of the 44th ACM tech. symp. on Computer science education (SIGCSE '13). ACM, New York, NY, USA, 41-46.

[4] D. Zingaro and L. Porter. 2014. Peer instruction: a link to the exam. In Proceedings of the 2014 conference on Innovation \& technology in computer science education (ITiCSE '14). ACM, New York, NY, USA, 255-260. 
[5] K. Beck and C. Andres. 2004. Extreme Programming Explained: Embrace Change. Addison-Wesley Professional.

[6] G. Braught, T. Wahls, and L. Marlin Eby. 2011. The Case for Pair Programming in the Computer Science Classroom. Trans. Computing Education 11, 1, Article 2 (February 2011)

[7] M. H. Goldwasser. 2002. A gimmick to integrate software testing throughout the curriculum, presented at the Proceedings of the 33rd SIGCSE Technical Symposium on Computer Science Education, ACM, New York, NY, pp. 271-275

[8] A. Gaspar, S. Langevin, N. Boyer, and R. Tindell. 2013. A preliminary review of undergraduate programming students' perspectives on writing tests, working with others, \& using peer testing. In Proceedings of the 14th annual ACM SIGITE conference. ACM, New York, NY, USA, 109-114.

[9] B. Means, M. Bakia, and R. Murphy. 2014. Learning online: What research tells us about whether, when and how. Routledge.
[10] S. Fincher, M. Petre. 2004. Computer Science Education Research. Taylor \& Francis. ISBN: 9026519699

[11] A.C. Graesser, D.F. Halpern, and M. Hakel. 2008. 25 principles of learning. Washington DC. Task Forces on Lifelong Learning at Work and at Home. Retrieved 10/10/2014 from https://activelearningps.files.wordpress.com/2014/07/25learning-principles-to-guide-pedagogy.pdf

[12] S. D. Craig, J. Sulling, A.Witherson, and B. Gholson. 2006. The deep-level reasoning effect: The role of dialogue and deeplevel-reasoning questions during vicarious learning. Cognition and Instruction 24, 565-591.

[13] A. Gaspar, S. Langevin, N. Boyer. 2007. Constructivist Apprenticeship through Antagonistic Programming Activities, Encyclopedia of Information Science and Technology, Vol. 2.

[14] C. Haller, V. Gallagher, T. Weldon, R. Felder. 2000. Dynamics of Peer Education in Cooperative Learning Workgroups. Journal of engineering education. 39:3, pp. $285-293$.

Table 5 - Q7 / Q12 - Items Comparisons

\begin{tabular}{|c|c|c|c|c|c|c|c|c|c|}
\hline \multirow[t]{2}{*}{ \# } & \multirow[t]{2}{*}{$\mathbf{Q}$} & \multicolumn{2}{|c|}{ Seldom } & \multicolumn{2}{|c|}{ Sometimes } & \multicolumn{2}{|c|}{ Often } & \multirow{2}{*}{$\chi^{2}$} & \multirow[t]{2}{*}{$\mathbf{p}$} \\
\hline & & F2F & ONL & F2F & ONL & F2F & ONL & & \\
\hline \multirow[t]{2}{*}{1} & 7 & 19 & 27 & 16 & 4 & 10 & 1 & 14.164 & .001 \\
\hline & 12 & 17 & 7 & 11 & 5 & 8 & 1 & 1.366 & .505 \\
\hline \multirow[t]{2}{*}{2} & 7 & 30 & 26 & 9 & 3 & 6 & 2 & 2.802 & .246 \\
\hline & 12 & 28 & 9 & 7 & 3 & 1 & 1 & 0.719 & $*$ \\
\hline \multirow[t]{2}{*}{3} & 7 & 18 & 21 & 23 & 8 & 4 & 3 & 5.596 & .061 \\
\hline & 12 & 8 & 3 & 17 & 6 & 11 & 4 & 0.006 & .997 \\
\hline \multirow[t]{2}{*}{4} & 7 & 9 & 15 & 14 & 10 & 22 & 7 & 7.957 & .019 \\
\hline & 12 & 10 & 5 & 15 & 6 & 11 & 2 & 1.230 & .541 \\
\hline \multirow[t]{2}{*}{5} & 7 & 17 & 25 & 20 & 3 & 8 & 4 & 13.616 & .001 \\
\hline & 12 & 17 & 8 & 16 & 4 & 3 & 1 & 0.826 & .662 \\
\hline \multirow[t]{2}{*}{6} & 7 & 26 & 21 & 13 & 8 & 5 & 3 & 0.336 & .845 \\
\hline & 12 & 12 & 1 & 11 & 4 & 13 & 8 & 3.808 & .149 \\
\hline
\end{tabular}

* Expected count is one, Chi-Square probably invalid

Table 6 - Q9 / Q14- Items Comparisons

\begin{tabular}{|c|c|c|c|c|c|c|c|c|c|}
\hline \multirow[t]{2}{*}{ \# } & \multirow[t]{2}{*}{$\mathbf{Q}$} & \multicolumn{2}{|c|}{ Seldom } & \multicolumn{2}{|c|}{ Sometimes } & \multicolumn{2}{|c|}{ Often } & \multirow[t]{2}{*}{$\chi^{2}$} & \multirow[t]{2}{*}{$\mathbf{p}$} \\
\hline & & F2F & ONL & F2F & ONL & F2F & ONL & & \\
\hline \multirow[t]{2}{*}{1} & 9 & 10 & 20 & 23 & 7 & 12 & 5 & 12.923 & .002 \\
\hline & 14 & 3 & 5 & 19 & 4 & 14 & 4 & 6.467 & .039 \\
\hline \multirow[t]{2}{*}{2} & 9 & 8 & 18 & 19 & 9 & 18 & 8 & 12.939 & .002 \\
\hline & 14 & 1 & 4 & 17 & 7 & 17 & 2 & 9.780 & .008 \\
\hline \multirow[t]{2}{*}{3} & 9 & 20 & 24 & 17 & 6 & 8 & 2 & 7.236 & .027 \\
\hline & 14 & 14 & 6 & 12 & 6 & 10 & 1 & 2.267 & .322 \\
\hline \multirow[t]{2}{*}{4} & 9 & 22 & 22 & 19 & 7 & 4 & 3 & 3.589 & .166 \\
\hline & 14 & 8 & 4 & 24 & 8 & 4 & 1 & 0.433 & .805 \\
\hline \multirow[t]{2}{*}{5} & 9 & 12 & 23 & 19 & 6 & 14 & 3 & 15.584 & .000 \\
\hline & 14 & 1 & 4 & 17 & 5 & 18 & 4 & 8.283 & .016 \\
\hline \multirow[t]{2}{*}{6} & 9 & 7 & 19 & 18 & 11 & 20 & 2 & 20.340 & .000 \\
\hline & 14 & 1 & 5 & 12 & 3 & 23 & 5 & 11.341 & .003 \\
\hline \multirow[t]{2}{*}{7} & 9 & 5 & 15 & 23 & 12 & 17 & 5 & 13.184 & .001 \\
\hline & 14 & 0 & 5 & 13 & 6 & 23 & 2 & 18.499 & .000 \\
\hline \multirow[t]{2}{*}{8} & 9 & 9 & 17 & 18 & 7 & 18 & 8 & 9.216 & .010 \\
\hline & 14 & 1 & 2 & 15 & 5 & 20 & 6 & 2.662 & .264 \\
\hline
\end{tabular}

